\title{
Nephrology
}

\section{Primary Hyperoxaluria Type 1 in Japan}

\author{
Tatsuya Takayama Masao Nagata Arata Ichiyama Seiichiro Ozono \\ Department of Urology, Hamamatsu University School of Medicine, Shizuoka, Japan
}

\section{Key Words}

Primary hyperoxaluria - Serine:pyruvate/alanine: glyoxylate aminotransferase - Alanine:glyoxylate aminotransferase

\begin{abstract}
Background/Aims: Current status of primary hyperoxaluria $(\mathrm{PH})$ has not been surveyed in Japan. Methods: Japanese patients with $\mathrm{PH}$ were reviewed in the published literature. Results: Fifty-nine patients were diagnosed as $\mathrm{PH}$ from 1962 to 2003 . The median ages both at diagnosis and at the onset of initial symptoms were 17 (range: 0.02-63) and 13 (range: 0-58) years, respectively. Twenty-nine (49\%) patients were older than 20 years at diagnosis, among whom 26 (90\%) already presented endstage renal failure (ESRF) or soon evolved into ESRF. Among 30 (51\%) diagnosed as $\mathrm{PH}$ under 20 years old, only $13(43 \%)$ were already in a terminal stage of renal insufficiency. Ten patients were diagnosed as $\mathrm{PH} 1$ by liver biopsy. We identified two types of enzymatic phenotypes in 3 of those patients examined. In 1 case, immunoreactive SPT/AGT protein level was very low due to accelerated proteolysis, while in other 2 cases, the immunoreactivity was detected on mitochondria due to mistargeting. Of 9 cases having been subjected to kidney transplantation at a median age of 20 years (range 7.340.0), it was only 2 cases that were reported to be successful, while the median survival time of the kidney
\end{abstract}

grafts being 1.4 years (range $0-7$ ). Of 4 patients having undergone combined liver/kidney transplantations (at the ages of 1.3, 1.4, 9 and 41 years, respectively), the surgery was successful in 3 cases; in the remaining one case, however, rejection required removal of the transplanted kidney was observed. The overall survival ratio of all the $59 \mathrm{PH}$ cases accounted for 77,71 and $55 \%$ at 5 , 10 and 20 years, respectively. Conclusion: Assuming that the majority of the 59 patients with $\mathrm{PH}$ reported was classified as $\mathrm{PH} 1$, it is postulated that morbidity of violent infantile $\mathrm{PH} 1$ in Japan might be less than those in the USA and Europe, and symptoms of elderly Japanese $\mathrm{PH} 1$ patients seem to be milder than those of Western patients. Establishment of an early detection system of $\mathrm{PH} 1$ and more popular application of combined liver/kidney transplantation deserve further study.

Copyright $(2005$ S. Karger AG, Basel

\section{Introduction}

Primary hyperoxaluria type 1 ( $\mathrm{PH} 1)$ is a rare autosomal-recessive inborn error of glyoxylate metabolism. It is characterized by a functional defect of the liver-specific enzyme serine:pyruvate/alanine:glyoxylate aminotransferase (SPT/AGT) which is localized in peroxisomes [1]. SPT/AGT is a unique enzyme characteristic of speciesspecific and food habit-dependent dual organelle localization; it is located entirely in peroxisomes in herbivores

\section{KARGER \\ Fax +4161306 1234 E-Mail karger@karger.ch} www.karger.com
C 2005 S. Karger AG, Basel 0250-8095/05/0253-0297\$22.00/0 www.karger.com/ajn
Dr. Tatsuya Takayama

Department of Urology, Hamamatsu University School of Medicine

1-20-1 Handayama

Hamamatsu, Shizuoka 431-3192 (Japan)

Tel. +81534352306, Fax +81534352305, E-Mail takayama@hama-med.ac.jp 
and humans, and largely in mitochondria in carnivores $[2,3]$. In herbivores and humans, a major source of glyoxylate, an immediate precursor of oxalate, is believed to be produced by oxidation of glycolate by glycolate oxidase in liver peroxisomes. Glycolate is an intermediate of photorespiration and thus is much higher in content in plants than in animal tissues [4]. Mitochondrial production of glyoxylate from hydroxyproline is assumed to play a significant role in carnivores [5], because the hydroxyproline content of collagen reaches about 10-13\% [6] and collagen accounts for about $30 \%$ of total animal protein. Although SPT/AGT is a bifunctional enzyme involved in the metabolism of both $L$-serine and glyoxylate, its contribution to $L$-serine metabolism is independent of mitochondrial or peroxisomal localization [7].

In the absence of functional SPT/AGT, oxalate and glycolate are produced in excess, leading to hyperoxaluria and hyperglycoric aciduria. The marked excess of oxalate leads to the formation of progressive renal deposition of calcium oxalate in the form of urolithiasis and/or nephrocalcinosis, followed by renal failure and systemic oxalosis, finally resulting in death usually before the third decade of age $[8,9]$.

PH1 demonstrates considerable phenotypic, enzymatic and genotypic heterogeneity [9-12]. A wide variety of enzymatic phenotypes have been identified in $\mathrm{PH} 1$, including loss of alanine:glyoxylate aminotransferase(AGT) catalytic activity due to inhibition of pyridoxal-phosphate binding [13, 14], loss of immunoreactive SPT/AGT protein due to accelerated degradation $[15,16]$, intraperoxisomal aggregation [17], and AGT mistargeting [18].

The clinical spectrum of primary hyperoxaluria $(\mathrm{PH})$ is very broad and patients are seen in all ages. Although some Japanese cases of PH1 were reported [19-23], PH1 has not adequately been surveyed in Japan. Japanese patients with $\mathrm{PH}$ reported in the literature were reviewed in the present investigation.

\section{Materials and Methods}

59 patients with $\mathrm{PH}$ could be seen reported from 1962 to 2003 in Japan. We reviewed those patients in the published literature as well as with personal communications.

\section{Results}

The male/female ratio was $35 / 24$ and, the median ages both at the initial symptoms and at diagnosis were 13 (range 0-58) and 17 (range 0.02-63) years, respectively.
The diagnostic workup included chemical analysis of oxalate and glycolate in the urine and serum (plasma), biopsy of liver, bone, and the other tissues, and clinical findings. The diagnosis criteria of $\mathrm{PH}$ in the chemical analysis stipulated urinary excretion of more than $100 \mathrm{mg}$ oxalate/day and $100 \mathrm{mg}$ glycolate/day or more than $100 \mu M$ oxalate in the serum measured by various methods. Following these chemical analyses, 17 patients were diagnosed as PH1. The liver biopsy was performed in 10 patients. Among them, 8 patients were diagnosed in our institution as PH1 by determination of liver serine:pyruvate aminotransferase (SPT) activity, while the phenotype of 3 patients was investigated. Based on the report that heavy deposition of oxalate in bones provides a reliable evidence for diagnosis of $\mathrm{PH} 1$ in hemodialysis patients [24], the bone biopsy and autopsy were performed in 12 patients each. Six patients were diagnosed as PH1 by both clinical findings and the calcium-oxalate crystal deposit in biopsy specimens of kidneys, arteries, or gingiva. The diagnostic workup for other two patients was unknown.

According to figure 1, about $40 \%$ of the total of 59 patients presented with the initial first signs of the disease before age 5 , besides about $75 \%$ of them being diagnosed as $\mathrm{PH}$ before that age. It is noteworthy that 29 patients $(49 \%)$ were older than 20 years old when diagnosis was made. It is also noted that in 22 cases (37\%), initial symptoms appeared after age 20, and as an extreme case, there was one patient who perceived the first sign of PH1 at the age of 58. There is a trend toward a longer interval between the ages at the first clinical symptoms and at diagnosis of $\mathrm{PH}$ when the onset occurred at higher ages.

Of the total 59 patients, 44 developed end-stage renal failure (ESRF), necessitating renal replacement therapy. Of these 44 patients with ESRF, 32 (73\%) showed nephrocalcinosis as the initial symptoms, evolving into subsequent onset of ESRF, and $16(50 \%)$ patients died during their respective follow-up periods (table 1).

Figure 2 demonstrates survival rate of the 59 patients after diagnosis as $\mathrm{PH}$. The overall survival rates were 77 , 71 and $55 \%$ at the time points after 5, 10 and 20 years, respectively. In 1986, PH1 was characterized as a congenital disease attributable to deficiency of liver peroxisomal AGT [1]. Thanks to our better understanding of the disease, prognosis of the patients has been greatly improved; for example, before 1986, 54\% of $\mathrm{PH}$ patient had died within 5 years after diagnosis, but after 1987 87\% of $\mathrm{PH}$ patients survived for at least 10 years.

Of 9 isolated kidney transplantations performed between 1982 and 1988, only 2 kidney grafts functioned 




Fig. 1. Age at first clinical manifestation and subsequent clinical courses of 59 cases of Japanese PH. Age at first clinical symptoms is indicated by a vertical bar and the age at diagnosis is shown by a filled circle. Dotted line and $\mathrm{D}$ represent renal replacement therapy and death, respectively.

well for 4.4 and 7 years, respectively. The median survival time of the kidney grafts was 1.4 years (range $0-7$ ). Table 2 shows profiles of 4 PH1 patients who underwent combined living-related liver/kidney transplantation at the ages of 1.3, 1.4, 9 and 41 years. With the exception of case 2, 3 patients were suffering from infantile or pediatric $\mathrm{PH} 1$. Outcomes of the cases 1, 2 and 3 were successful, and they are still alive. However, case 3 needed a kidney graftectomy due to acute rejection. In each case, the liver/ kidney transplantation was performed after treatment by hemodialysis or continuous ambulatory peritoneal dialysis (CAPD). Pre-emptive liver transplantation has not been performed. Cadaveric liver transplantation has not also been performed due to the complex feelings of Japanese people toward the departed.

SPT/AGT exhibits not only the AGT activity but also the SPT activity with both of these two activities being of physiological significance [7]. Assay of the AGT activity
Table 1. Outcome in relation to initial symptoms

\begin{tabular}{lcc}
\hline Initial symptoms & Patients (\%) & ESRF (\%)* \\
\hline NC & $16(27)$ & $16 / 16(100)$ \\
NC with UL & $18(31)$ & $16 / 18(89)$ \\
UL & $14(24)$ & $9 / 14(64)$ \\
No NC, no UL & $3(5)$ & $0 / 3(0)$ \\
Unknown & $8(13)$ & $3 / 8(38)$ \\
Total number & 59 & 44 \\
\hline
\end{tabular}

* ESRF at diagnosis and that developed during the follow-up period. $\mathrm{NC}=$ Nephrocalcinosis; $\mathrm{UL}=$ urolithiasis. 

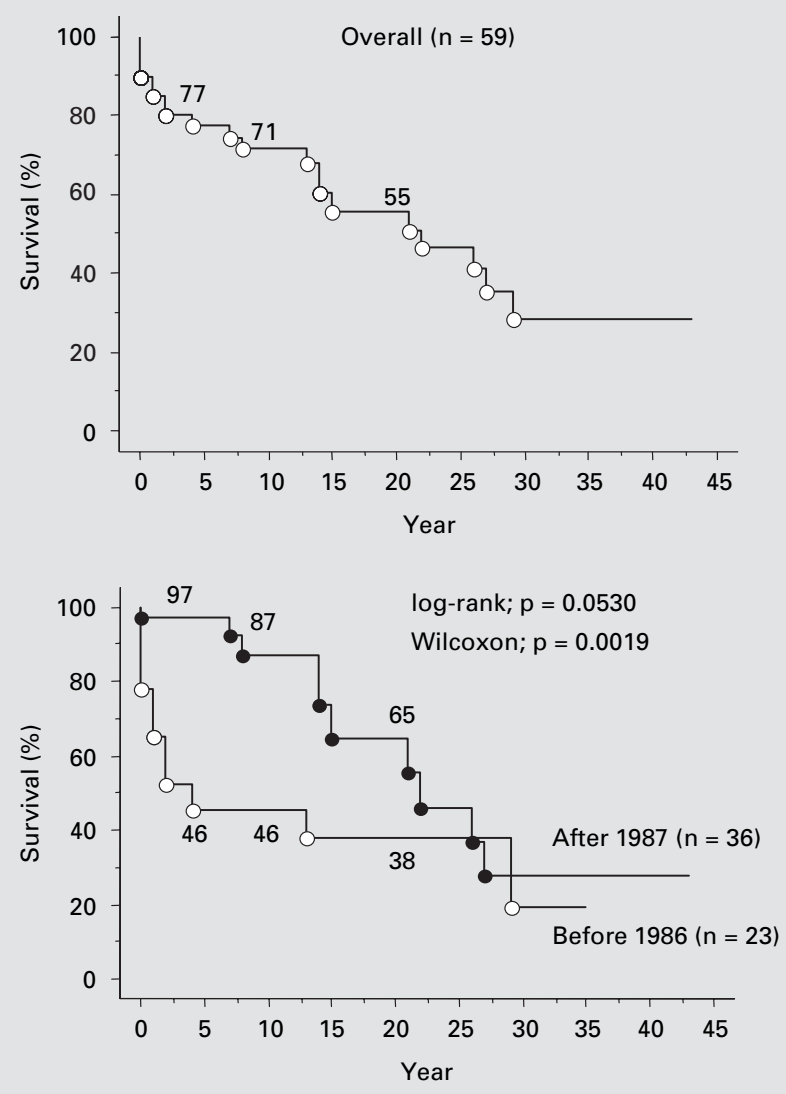

Fig. 2. Survival after diagnosis as $\mathrm{PH}$.

of SPT/AGT in human liver extract is associated with a problem that glutamate:glyoxylate aminotransferase (GGT) also catalyzes transamination between alanine and glyoxylate. Therefore, the GGT activity should be concomitantly assayed, followed by appropriate correction of the apparent AGT activity with use of the activity catalyzed by GGT, assuming the AGT activity of GGT to be $66 \%$ of its GGT activity [25]. In contrast, however, the SPT activity in human liver extract is almost entirely catalyzed by SPT/AGT, and this is the reason why we prefer assay of SPT/AGT with its SPT activity [26, 27]. In fact, only a negligible SPT activity was detectable in liver homogenate from some PH1 patients. Determination of the liver SPT activity as mentioned above permitted us to diagnose 8 patients as $\mathrm{PH} 1$, whereas phenotypes of only 3 cases have so far been clarified in detail. In one case with a Ser to Pro substitution at residue 205 due to a $\mathrm{T}$ to $\mathrm{C}$ point mutation, AGT level was very low in terms of not only the activity but also the protein to be detected on Western blot and immunocytochemical analysis; however, of interest to note is the fact that the level of translatable AGT-mRNA was even higher than normal. The SPT/AGT defect in this case was then found to be ascribable to an accelerated proteolysis, but unfortunately the proteolytic system responsible for degradation of the mutant SPT/AGT has not been identified $[15,16]$. In the other two cases, mistargeting of SPT/AGT to mitochondria was evidenced by immnocytochemical analysis [pers. commun., with permission of the physicians in charge].

Table 2. Combined living-related liver/ kidney transplantation

\begin{tabular}{|c|c|c|c|c|}
\hline Case & Symptom & Liver biopsy & Dialysis & Outcome \\
\hline 1 & NC (2 months) & SPT (-) & 4 months (CAPD) & 5 years, alive \\
\hline 1.3 years, $F$ & Uox $\uparrow(2$ months $)$ & & & \\
\hline 2 & $\mathrm{NC}$ (3 years) & - & 33 years (HD) & 1.5 years, alive \\
\hline 41 years, $M$ & Uox $\uparrow$ (41 years) & & & \\
\hline 3 & NC (6 months) & SPT (-) & 8 years (HD) & kidney graft loss* \\
\hline 9 years, $M$ & Uox $\uparrow$ ( 2 years) & & & \\
\hline 4 & NC (6 months) & SPT (-) & 8 months (CAPD) & 9 months, alive \\
\hline 1.4 years, $M$ & Uox $\uparrow$ (6 months) & & & \\
\hline
\end{tabular}

* The kidney graftectomy was performed 3 weeks after kidney transplantation due to rupture of the graft by rejection.

NC = Nephrocalcinosis; Uox = urinary oxalate excretion; SPT = serine:pyruvate transaminase; $\mathrm{HD}=$ hemodialysis; $\mathrm{CAPD}=$ continuous ambulatory peritoneal dialysis. 


\section{Discussion}

We reviewed 59 Japanese patients reported in the published literature between 1962 and 2003, as well as personal communications. The definitive diagnosis of $\mathrm{PH} 1$ requires measurement of the SPT/AGT activity in liver biopsy, but only 10 of 59 cases were successfully diagnosed by liver biopsy. Recently, Rumsby et al. [28] reported that a preliminary screening for limited mutations in AGTX genes could play a useful role as the first line investigation for diagnosis of $\mathrm{PH} 1$ with less risk being entailed than did the liver biopsy.

Latta and Brodehl [9] reviewed 330 European cases of PH1 in 1990. Accordingly, we compared the 59 cases of Japanese PH with the 330 European cases of PH1 and the results are shown in table 3. In Japan, as is obvious from table 3 , less $\mathrm{PH}$ patients perceived initial symptoms before age 5 , whereas as high as $92 \%$ of the $\mathrm{PH}$ patients were symptomatic after age 25 . The first symptoms consisted of urolithiasis in $64 \%$ of the Western cases, and $55 \%$ in Japanese. Regrettably, $75 \%$ of $\mathrm{PH}$ patients $(93 \%$ in case of late-onset $\mathrm{PH}$ ) were already uremic status (ESRF) at diagnosis. Small number of cases required renal replacement therapy by the end of 3rd decade in Japan, partly because of involvement of late-onset PH1. van Woerden et al. [12] reported that clinical symptoms of adult PH1 were generally milder than infantile or pediatric PH1. It is conceivable that in late-onset PH1, the milder signs and symptoms prior to the development of ESRF have not been adequately recognized, whereby the opportunity for a timely diagnosis has been missed. Given that many late-onset PH cases insidiously progressed to the stage of ESRF at diagnosis or soon followed by ESRF (fig. 1), we believe that screening for PH1 should be performed in all the patients with ESRF irrespective of its origin.

In this study, all the $\mathrm{PH}$ patients with nephrocalcinosis developed ESRF (table 1). It has also been described that the progression to renal insufficiency was associated with complication of nephrocalcinosis [9-12]. Pyridoxine-responsive PH1 has been documented; in Japan, however, only 2 cases were reported to be responsive to pyridoxine.

Although studies of PH in Japan are not yet advanced, it is evident that the morbidity of violent infantile PH1 appeared to be less than that in the West, and that symptoms of late-onset Japanese PH1 patients seemed to be milder than those of Western patients. The reason why Japanese PH patients suffer from apparently milder symptoms is not clear, but it may be related, at least in
Table 3. Comparison with PH1 in the West and in Japan

\begin{tabular}{lll}
\hline & $\begin{array}{l}\text { Latta and } \\
\text { Brodehl [9] }\end{array}$ & Japan \\
\hline Initial symptoms before age 5, \% & 54 & 42 \\
Symptomatic before age 25, \% & 92 & 71 \\
First signs indicative of UL, \% & 64 & 55 \\
Already uremic at diagnosis, \% & 14 & 75 \\
$\begin{array}{l}\text { Required renal replacement therapy } \\
\text { by the end of 3rd decade, \% }\end{array}$ & 80 & 77 \\
\hline
\end{tabular}

$\mathrm{UL}=$ Urolithiasis.

part, to the food habits of Japanese. This hypothesis could be substantiated by the findings that the elderly Japanese especially prefer boiled greens rather than fried or raw vegetables [20].

According to our preliminary experiment, cooking of spinach in hot water reduced the oxalate content to about half [unpubl. data]. In clinical settings, we should establish an early detection system of $\mathrm{PH} 1$ and popularization of combined liver/kidney transplantation. As a natural consequence, organization of a PH registry system is an urgent issue for nephrologists, urologists and pediatricians.

\section{Acknowledgements}

The authors thank Dr. Yoshihide Ogawa, Department of Urology, University of the Ryukyus and Dr. Kouji Shiraga, Department of Pediatrics, Saitama Saiseikai Hospital for his useful discussion. 


\section{References}

1 Danpure CJ, Jennings PR: Peroxisomal alanine:glyoxylate aminotransferase deficiency in primary hyperoxaluria type I. FEBS Lett 1986; 201:20-24.

-2 Noguchi T, Takada Y: Peroxysomal localization of serine:pyruvate aminotransferase in human liver. J Biol Chem 1978;253:7598-7600.

$\checkmark 3$ Danpure CJ, Guttridge KM, Fryer P, Jennings PR, Allsop T, Purdue PE: Subcellular distribution of hepatic alanine:glyoxylate aminotransferase in various mammalian species. Cell Sci 1990;97:669-678.

4 Harris KS, Richardson KE: Glycolate in the diet and its conversion to urinary oxalate in the rat. Invest Urol 1980;18:106-109.

5 Takayama T, Fujita K, Suzuki K, Sakaguchi M, Fujie M, Nagai E, Watanabe S, Ichiyama A, Ogawa Y: Control of oxalate formation from $L$-hydroxyproline in liver mitochondria. J Am Soc Nephrol 2003;14:939-946.

6 Neuman RE, Logan MA: The determination of hydroxyproline. J Biol Chem 1950;184: 299-306.

$>7$ Xue HH, Sakaguchi T, Fujie M, Ogawa H, Ichiyama A: Flux of the $L$-serine metabolism in rabbit, human, and dog livers: substantial contributions of both mitochondrial and peroxisomal serine:pyruvate/alanine:glyoxylate aminotransferase. J Biol Chem 1999;274: 16028-16033.

8 Danpure CJ, Purdue PE: Primary hyperoxaluria; in Scriver CR, Beaudet AL, Sly WS, Valle D (eds): The Metabolic and Molecular Bases of Inherited Disease, ed 7. New York, McGraw-Hill, 1995, pp 2385-2424.

$\checkmark 9$ Latta K, Brodehl J: Primary hyperoxaluria type I. Eur J Pediatr 1990;149:518-522.

$\checkmark 10$ Kopp N, Leumann E: Changing pattern of primary hyperoxaluria in Switzerland. Nephrol Dial Transplant 1995;10:2224-2227.

11 Milliner DS, Wilson DM, Smith LH: Clinical expression and long-term outcomes of primary hyperoxaluria types 1 and 2. J Nephrol 1998; 11(suppl 1):56-59.
12 van Woerden CS, Groothoff JW, Wanders RJ, Davin JC, Wijburg FA: Primary hyperoxaluria type 1 in The Netherlands: prevalence and outcome. Nephrol Dial Transplant 2003;18:273279.

13 Purdue PE, Lumb MJ, Allsop J, Minatogawa Y, Danpure CJ: A glycine-to-glutamate substitution abolishes alanine:glyoxylate aminotransferase catalytic activity in a subset of patients with primary hyperoxaluria type 1 . Genomics 1992;13:215-218.

14 Lumb MJ, Danpure CJ: Functional synergism between the most common polymorphism in human alanine:glyoxylate aminotransferase and four of the most common disease-causing mutations. J Biol Chem 2000;275:3641536422.

15 Nishiyama K, Funai T, Katafuchi R, Hattori F, Onoyama K, Ichiyama A: Primary hyperoxaluria type I due to a point mutation of $\mathrm{T}$ to $\mathrm{C}$ in the coding region of the serine:pyruvate aminotransferase gene. Biochem Biophys Res Commun 1991;176:1093-1099.

16 Nishiyama K, Funai T, Yokota S, Ichiyama A: ATP-dependent degradation of a mutant serine: pyruvate/alanine:glyoxylate aminotransferase in a primary hyperoxaluria type 1 case. J Cell Biol 1993;123:1237-1248.

17 Danpure CJ, Purdue PE, Fryer P, Griffiths S, Allsop J, Lumb MJ, Guttridge KM, Jennings PR, Scheinman JI, Mauer SM, Davidson NO Enzymological and mutational analysis of a complex primary hyperoxaluria type 1 phenotype involving alanine:glyoxylate aminotransferase peroxisome-to-mitochondrion mistargeting and intraperoxisomal aggregation. Am J Hum Genet 1993;53:417-432.

18 Danpure CJ, Cooper PJ, Wise PJ, Jennings PR: An enzyme trafficking defect in two patients with primary hyperoxaluria type 1:peroxisomal alanine/glyoxylate aminotransferase rerouted to mitochondria. J Cell Biol 1989; 108:1345-1352.
19 Kazama-Saegusa S, Kazama JJ, Sugaya H, Takamiya H, Terano A, Ichiyama A: A case of late onset primary hyperoxaluria type I (PH-I) presented with black liver. Clin Nephrol 1998; 50:184-187.

20 Ichiyama A, Oda T, Maeda-Nakai E: Primary hyperoxaluria type 1 in Japan. Cell Biochem Biophys 2000;32:171-176.

21 Matsumura Y, Miyachi Y, Yamamoto H, Hayashi M, Egawa H, Tanaka K: Calcinosis cutis in a patient with primary hyperoxaluria due to hepatic enzyme deficiency. J Dermatol 2001;28:578-579.

22 Sato S, Fuchinoue S, Kimikawa M, Tojimbara T, Nakajima I, Teraoka S, Shiraga H, Ito K: Sequential liver-kidney transplantation from a living-related donor in primary hyperoxaluria type 1 (oxalosis). Transplant Proc 2003;35: 373-374.

23 Inoue $\mathrm{Y}$, Masuyama $\mathrm{H}$, Ikawa $\mathrm{H}$, Mitsubuchi $\mathrm{H}$, Kuhara T : Monitoring method for pre- and post-liver transplantation in patients with primary hyperoxaluria type I. J Chromatogr [B] 2003;792:89-97.

24 Fukushima M, Aihara Y, Ichiyama A: Immunochemical studies on induction of rat liver mitochondrial serine:pyruvate aminotransferase by glucagons. J Biol Chem 1978;253:11871194.

25 Ishikawa K, Kaneko E, Ichiyama A: Pyridoxal 5 '-phosphate binding of a recombinant rat serine:pyruvate/alanine:glyoxylate aminotransferase. J Biochem 1996;119:970-978.

26 Mathews M, Stauffer M, Cameron EC, Maloney N, Sherrard DJ: Bone biopsy to diagnose hyperoxaluria in patients with renal failure. Ann Intern Med 1979;90:777-779.

-27 Thompson JS, Richardson KE: Isolation and characterization of a glutamate-glycine transaminase from human liver. Arch Biochem Biophys 1966;117:599-603.

28 Rumsby G, Williams E, Coulter-Mackie M: Evaluation of mutation screening as a first line test for the diagnosis of the primary hyperoxalurias. Kidney Int 2004;66:959-963. 